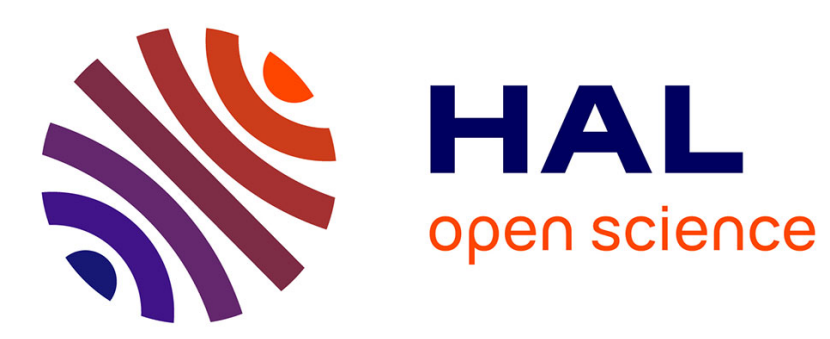

\title{
Les pharyngotomies latérales
}

\author{
O. Laccourreye, A. Villeneuve, F. Rubin, C.F. Holsinger
}

\section{To cite this version:}

O. Laccourreye, A. Villeneuve, F. Rubin, C.F. Holsinger. Les pharyngotomies latérales. Annales françaises d'Oto-rhino-laryngologie et de Pathologie Cervico-faciale, 2019, 136, pp.135 - 138. 10.1016/j.aforl.2018.08.001 . hal-03486487

\section{HAL Id: hal-03486487 \\ https://hal.science/hal-03486487}

Submitted on 20 Dec 2021

HAL is a multi-disciplinary open access archive for the deposit and dissemination of scientific research documents, whether they are published or not. The documents may come from teaching and research institutions in France or abroad, or from public or private research centers.
L'archive ouverte pluridisciplinaire HAL, est destinée au dépôt et à la diffusion de documents scientifiques de niveau recherche, publiés ou non, émanant des établissements d'enseignement et de recherche français ou étrangers, des laboratoires publics ou privés.

\section{다)(1) $(5$}

Distributed under a Creative Commons Attribution - NonCommerciall 4.0 International 


\section{Les pharyngotomies latérales.}

Ollivier Laccourreye (1)*, Alexandre Villeneuve (1), François Rubin (1), Christopher F. Holsinger (2).

Services d'Otorhinolaryngologie et de Chirurgie cervico-faciale

(1) Université Paris Descartes Sorbonne Paris Cité, HEGP, APHP, Assistance Publique des Hôpitaux de Paris, Paris, France

(2) Department of Otorhinolaryngology Head Neck Surgery, Stanford University, Palo Alto, CA, USA.

\section{* Auteur correspondant :}

E-mail : ollivier.laccourreye@aphp.fr (Ollivier Laccourreye)

Service ORL, HEGP, 20-40 rue Leblanc, 75015 Paris, France

\section{Déclaration d'intérêts}

Les auteurs signalent ne pas avoir de conflits d'intérêt

\section{Résumé.}

Dans cette note technique, les auteurs précisent, au vu des données de la littérature médicale scientifique, les points techniques clefs, les variantes, les erreurs à éviter, ainsi que les principaux résultats fonctionnels des pharyngotomies latérales dans les cancers dont le point de départ se situe au niveau de l'oro et/ou de l'hypopharynx.

Mots clefs. pharyngotomie, oropharynx, hypopharynx, cancer. 


\section{Introduction.}

Au sens étymologique du terme, la pharyngotomie latérale est la section de la paroi latérale du pharynx. Cette voie d'abord, décrite en 1929 par le chirurgien britannique William Trotter (Figure 1) [1] puis modifiée par divers auteurs, a été proposée, en cancérologie cervicofaciale, pour réaliser l'exérèse de tumeurs à point de départ hypopharyngé (paroi postérieure de l'hypopharynx, paroi pharyngée latérale, face externe du sinus piriforme) ou oropharyngé (base de la langue latérale, région sous-amygdalienne, sillon amygdalo-glosse) [2-14]. Avec la recrudescence des cancers épidermoides de l'oropharynx en rapport avec l'infestation par l'human papilloma virus survenant à un âge plus précoce et la connaissance des séquelles de la radiothérapie, cette approche chirurgicale qui préserve l'intégrité mandibulaire connaît un regain d'intérêt, depuis une dizaine d'années [5-16]. Dans cette note technique, les auteurs détaillent les points techniques clefs et les variantes de cet abord du pharynx ainsi que les erreurs à éviter et les résultats fonctionnels publiés.

\section{Technique.}

Une fois le patient intubé et une sonde naso-gastrique insérée, une trachéotomie est réalisée, par incision horizontale en regard du 3-4è anneau trachéal, séparée de la cervicotomie menée de la pointe de l'apophyse mastoïde au triangle de Sedillot, un centimètre en arrière au bord antérieur du muscle sternocléidomastoïdien ipsilatéral.

L'exposition du champ opératoire débute après réalisation de l'évidement ganglionnaire jugulo-carotidien ipsilatéral adapté au statut ganglionnaire pré opératoire. Les points de repères anatomiques sont: la grande corne de l'os hyoïde, la grande corne du cartilage thyroïde, l'axe carotidien avec la naissance des artères linguale et thyroïdiennes 
supérieures et le bord externe des muscles sous hyoïdiens (Figure 2). Le tronc du nerf grand hypoglosse est repéré au-dessus de la grande corne de l'os hyoïde. Le tronc de l'artère thyroïdienne supérieure est repéré à son origine au niveau du bulbe carotidien ou de l'artère carotide externe (Figure 2). Le nerf laryngé supérieur est repéré en dedans de la bifurcation entre le tronc de l'artère carotide externe et la naissance de la seconde branche de l'artère carotide externe : l'artère linguale (Figure 2). Le tronc du nerf laryngé supérieur réalise à ce niveau, dans la profondeur, la bissectrice entre ces deux artères pour, après un court trajet en dedans, rejoindre les éléments vasculaires laryngés supérieurs qu'il aborde avant de pénétrer dans la loge hyothyroépiglottique (Figure 2).

La principale difficulté des pharyngotomies latérales tient au choix du point d'entrée dans le pharynx. Celui ci doit être à distance suffisante de la tumeur, pour permettre une résection sous contrôle de la vue avec des limites de résection saines. Cette « entrée » dans le pharynx en «zone saine» est grandement facilitée par le tatouage (à l'encre) profond, de la périphérie muqueuse et musculaire de la zone à réséquer, lors de l'endoscopie à visée diagnostique. En fonction de la topographie tumorale et de sa localisation « en hauteur », quatre éléments barrent l'accès: le nerf grand hypoglosse et le nerf laryngé supérieur, l'artère linguale et l'artère thyroïdienne supérieure (Figure 2). Le repérage et la préservation des deux nerfs sus cités isolés et mis en pont (Figure 3), avant le temps d'ouverture, est une des clefs pour des suites opératoires simples. La préservation du tronc de l'artère linguale n'est pas obligatoire mais, si l'exérèse tumorale nécessite la résection de base de langue ipsilatérale, la préservation de cette artère offre la possibilité de réaliser un lambeau de translation de langue mobile (set-back tongue flap [17]). La section du tronc de l'artère laryngée supérieure est classiquement réalisée, associée à celle du tronc de l'artère thyroïdienne supérieure (Figures 3-5), pour exposer largement la paroi pharyngée à hauteur du larynx. Cependant, si un 
lambeau pédiculé des muscles sous-hyoïdiens ipsilatéraux est envisagé lors du temps de fermeture, le pédicule vasculaire thyroïdien supérieur doit être impérativement conservé et alors seul le pédicule vasculaire laryngé supérieur sera sectionné [18].

Au terme du temps d'exposition sus décrit, schématiquement, trois situations différentes conditionnant l'abord existent :

- En présence de tumeurs développées au niveau de la paroi externe du sinus piriforme et/ou de la paroi pharyngée latérale (pilier postérieur de l'amygdale), le point d'entrée le plus sûr se situe au niveau de la partie postérieure de la vallécule ipsilatérale. Pour y accéder en toute facilité, il convient de compléter l'exposition en désinsérant au bistouri électrique tendon du muscle digastrique, muscles et ligaments stylo-hyoïdiens, muscle mylo-hyoïdien et muscles sous-hyoïdiens respectivement du bord supérieur et du bord inférieur de la grande corne de l'os hyoïde puis de sectionner la grande corne de l'os hyoïde en arrière de la petite corne (Figures 3, 4). La traction vers l'arrière de la grande corne de l'os hyoïde permet de visualiser la muqueuse de la vallécule immédiatement sus jacente à la partie externe de la graisse de la loge hyothyroépiglottique. L'incision horizontale de la muqueuse, vers l'arrière, permet de visualiser la topographie exacte de la tumeur. Les muscles sous hyoïdiens, sont alors décollés de la loge hyothyroépiglottique puis séparés du tiers postérieur de la face externe de la lame cartilagineuse thyroïdienne en incisant le périchondre externe horizontalement au bord supérieur du cartilage thyroïde et verticalement en avant de l'insertion des muscles constricteurs inférieurs (Figure 5). Le tiers postérieur de l'aile cartilagineuse thyroïdienne (qui correspond à la projection du sinus piriforme) est ensuite incisé à la scie oscillante réalisant un triangle cartilagineux à base supérieure dont la pointe correspond au bord inférieur de la pharyngotomie latérale et se situe à hauteur de la petite corne préalablement sectionnée (Figure 5). A la partie basse de cette exposition, la jonction 
entre le bord inférieur de l'aile thyroïdienne et la petite corne est sectionnée à distance du tronc du nerf laryngé inférieur et de l'insertion des fibres du muscle crico-thyroïdien ipsilatéral (Figures 5). En avant, la paroi pharyngée du sinus piriforme est sectionnée le long de la section cartilagineuse de l'aile cartilagineuse. Lors de cette exérèse, le tronc du nerf laryngé supérieur préalablement isolé est épargné et passe en pont en dehors de la pièce opératoire (Figures 4,5). Puis, sous contrôle de la vue, l'exérèse est menée en incisant les muscles constricteurs inférieurs verticalement en arrière du bord postérieur de la lame cartilagineuse thyroïdienne ipsilatérale (Figure 5).

- En présence d'une tumeur à point de départ basi-lingual et/ou amygdalo-glosse, le ventre postérieur du digastrique, le muscle et ligament stylo-hyoïdien sont sectionnés de la petite corne de l'os hyoïde et réclinés vers l'arrière visualisant l'insertion du muscle styloglosse dans la base de langue (Figures 3,4). L'artère faciale est isolée, sectionnée et liée à son entrée à la partie postérieure de la loge sous maxillaire ce qui permet de refouler la glande sous maxillaire ipsilatérale vers l'avant (Figures 3,4). L'insertion du muscle mylo-hyoidien sur le tiers postérieur de la grande corne de l'os hyoïde est sectionnée (Figures 3,4). La première branche de l'artère linguale, issue de son bord supérieur, est liée et sectionnée (Figure 4). La pharyngotomie latérale est alors réalisée au bord postérieur de la grande corne de l'os hyoïde au bistouri froid lame 11 sur un centimètre au travers des fibres du muscle constricteur moyen. L'os hyoïde est alors sectionné à l'union petite corne - grande corne. La pharyngotomie latérale est prolongée horizontalement, en dedans, au bord inférieur de la grande corne de l'os hyoïde. Ce geste permet de visualiser la totalité de la base de langue et du sillon amygdalo-glosse ipsilatéral. Ce temps peut nécessiter l'isolement du tronc du XII ipsilatéral qui sera isolé et préservé en pont comme le nerf laryngé supérieur (Figures 3,4). Lorsque la visualisation est limitée, le champ opératoire peut être agrandi, soit en réséquant partiellement l'angle mandibulaire [8], soit en incisant en avant le plancher buccal après avoir 
réalisé un évidement de la zone Ib ipsilatérale et en connectant l'incision ainsi réalisée dans la cavité buccale avec la pharyngotomie proprement dite [9]. Cet abord peut aussi être très facilement combiné à sa partie haute (à hauteur du muscle styloglosse) avec une oropharyngectomie latérale trans orale [19], si la loge amygdalienne doit être réséquée pour obtenir des limites de résection saines.

- En présence d'une tumeur de la paroi pharyngée postérieure, il n'est pas nécessaire de sectionner la grand corne de l'os hyoïde ni de désinsérer les muscles sus et sous hyoïdiens de l'os hyoïde. En utilisant un crochet placé sur l'extrémité de la grande corne du cartilage thyroïde, l'aide exerce une traction qui rote et ascensionne le larynx. La pharyngotomie latérale est alors réalisée au bord postérieur de la grande corne du cartilage thyroïde au bistouri froid lame 11. Le risque, lors de ce temps opératoire, est de se perdre dans les fibres du muscle constricteur inférieur. Aussi, il convient de ponctionner franchement ce plan musculaire bien perpendiculairement à lui et en utilisant toute la longueur de la lame du bistouri. Sous contrôle de la vue, la pharyngotomie latérale est ensuite agrandie sur la hauteur nécessaire pour visualiser la totalité de la paroi pharyngée postérieure atteinte tout en respectant nerf grand hypoglosse, artère linguale et tronc du nerf laryngé supérieur, ce dernier élément étant alors le plus souvent, le seul à être isolé (Figure 6). La paroi pharyngée postérieure est alors décollée du plan pré vertébral puis tractée pour permettre une résection à distance de la tumeur tout en préservant l'intégrité du nerf laryngé supérieur.

En dehors de la pharyngotomie latérale réalisée pour tumeur de la paroi pharyngée postérieure, la fermeture s'effectue soit par simple rapprochement de la paroi pharyngée restante suturée de haut en bas à la base de langue, à la vallécule et à la paroi interne du sinus piriforme recouvert en utilisant les muscles sous-hyoïdiens ipsilatéraux préservés, soit, si la fermeture par simple rapprochement est impossible, en utilisant divers lambeaux locaux, à 
distance pédiculés, voire libres $[6,9-11,13,17,18]$. En présence d'une tumeur de la paroi pharyngée postérieure et en fonction de la taille du défect, trois attitudes sont possibles au niveau la zone réséquée: le décollement du plan vertébral de l'extrémité restantes de la paroi pharyngée postérieure et sa suture à la paroi pharyngée latérale restante, la cicatrisation dirigée par première intention après suture de la paroi pharyngée latérale conservée à l'aponévrose pré vertébrale ou la mise en place d'un lambeau.

L'intervention se termine avec la réalisation des temps conventionnels vérification de l'hémostase, mise en place d'un drainage, fermeture sous-cutanée et cutanée, mise en place d'une canule à ballonnet et pansement non compressif.

\section{Discussion.}

Les pharyngotomies latérales «modernes» se distinguent de la voie d'abord décrite en 1929 par William Trotter [1] (Figure 1) par l'adaptation de la topographie de cette incision trans pharyngée à la topographie tumorale, au mieux guidée par le tatouage des limites à réséquer lors de l'endoscopie à visée diagnostique, mais aussi et surtout par le maintien de l'intégrité du tronc des nerfs grand hypoglosse et laryngé supérieur ipsilatéraux et le respect de la continuité osseuse mandibulaire.

Le maintien de l'intégrité des nerfs grand hypoglosse et laryngé supérieur ipsilatéraux est reconnu comme un point clef lors de la déglutition car ces éléments coordonnent la sensation et la propulsion du bolus alimentaire au sein de l'oropharynx et de l'hypopharynx avec l'occlusion laryngée [20]. La lecture des travaux consacrés aux résultats fonctionnels après pharyngotomie latérale pour cancer à point de départ pharyngé souligne le bénéfice tiré de la préservation de ces structures nerveuses. Ainsi, dans la plus large cohorte publiée, constituée de 91 patients avec un cancer épidermoide invasif de l'oropharynx latéral opérés 
par pharyngotomie latérale et suivis au moins 5 ans ou jusqu'au décès, les auteurs font état en post-opératoire de la survenue de troubles sévères de la déglutition et de pneumopathies d'inhalation respectivement seulement dans $6,3 \%$ et $4,3 \%$ de cas pour une seule gastrostomie permanente[13]. Dans cette même étude, les auteurs soulignent la rareté des fistules salivaire notées dans $4,3 \%$ des cas mais aussi la gravité de cette complication responsable du seul décès péri opératoire d'origine chirurgicale [13]. La similitude avec les résultats notés si la pharyngotomie est placée au niveau de l'hypopharynx $[6,7,12]$ ou est très étendue combinant incision du pharynx, de l'hypopharynx et du plancher buccal voire résection trans orale de la région amygdalienne[8-11] souligne l'importance des temps opératoire qui visent à repérer, isoler et préserver le tronc de ces deux nerfs (Figures 2-4). Il apparaît aussi que la réalisation d'un lambeau lors du temps de fermeture ne pénalise pas les résultats fonctionnels, tant qu'une hémi base de langue mobile est conservée [9-12] et dans ce cadre l'intérêt du lambeau dénommé « set back tongue flap » (qui impose la préservation du tronc de l'artère linguale ipsilatérale) décrit en 1980 par Schechter et al.[17] doit être souligné. Enfin, les résultats fonctionnels des pharyngotomies latérales en terme de déglutition soutiennent largement la comparaison avec les résultats de la radio chimiothérapie [21,22]. La préservation de l'intégrité mandibulaire est aussi un point très important en faveur des pharyngotomies latérales «modernes»; la lecture de la littérature médicale scientifique souligne le caractère néfaste, sur le plan fonctionnel, de la mandibulotomie dans le traitement chirurgical « conservateur » des cancers de l'oropharynx avec la survenue de complications locales sévères une fois sur cinq, sans compter les problèmes dentaires, les défauts d'ostéosynthèse, la limitation de l'ouverture buccale et les malocclusions, tous éléments dont la survenue et la sévérité sont favorisées par la réalisation d'une radiothérapie post opératoire [23,24]. Il convient enfin de savoir que la résection de l'angle mandibulaire, 
sans mandibulotomie, telle que décrite par Cocek et al.[8] pour améliorer la visualisation des limites tumorales, ne semble pas générer les complications induites par la mandibulotomie.

Remerciements: Les auteurs remercient l'association Progrès 2000 pour son soutien technique. 


\section{Références.}

1 Trotter W. Operations for malignant disease of the pharynx. British Journal of Surgery 1929;16:485-495.

2 Harris PF, Rosenfeld L, Ward PH. Lateral pharyngotomy approach for lesions of the base of the tongue, pharynx and larynx. South Med J 1968; 61:1276-80.

3 Stern SJ. Anatomy of the lateral pharyngotomy approach. Head Neck 1992:14: 153-156.

4 Byers R.M. Anatomic correlates in head neck surgery. The lateral pharyngotomy. Head Neck 1994;16:460-463.

5 Ogura JH, Watson RK, Jurema AA. Partial pharyngectomy and neck dissection for posterior hypopharyngeal cancer. Immediate reconstruction with preservation of voice. Laryngoscope. 1960;70:1523-1534.

6 Steinhart H, Constantinidis J, Iro H. Larynx preserving surgery in carcinomas of the posterior hypopharyngeal wall by reconstruction with a free flap. HNO 1998; 46:135-139.

7 Holsinger FCh, Motamed M, Garcia D, Brasnu D, Ménard M, Laccourreye O. Resection of selected invasive squamous cell carcinoma of the piriform sinus mean of the lateral pharyngotomy approach: the partial lateral pharyngectomy. Head Neck 2006;28:705-711.

8 Cocek A, Pru I, Hahn C, Hahn A. Lateral pharyngotomy extended by resection of the mandibular angle an alternative approach to the surgical treatment of oropharyngeal (tonsillar) cancers. Acta Oto-Laryngologica 2009;129:318-323

9 Laccourreye O, Seccia V, Ménard M, Garcia D, Vacher Ch, Holsinger FCh. Extended lateral pharyngotomy for selected squamous cell carcinoma of the lateral tongue base. Ann Otol Rhinol Laryngol 2009;118:428-434. 
10 Bozec A, Poissonnet G, Chamorey E, et al. Transoral and cervical approach without mandibulotomy for oropharynx cancer with fasciocutaneous radial forearm free flap reconstruction. Ann Otolaryngol Chir Cervicofac 2009;126:182189.

11 Masuda M, Fukushima J, Kadota H, Kamizono K, Ejima M, Taura M. Mandible preserving pull-through oropharyngectomy for advanced oropharyngeal cancer: a pilot study. Auris Nasus Larynx 2011;38:392-397.

12 Lim YC, Jeong HM, Shin HA, Choi EC. Larynx-preserving partial pharyngectomy via lateral pharyngotomy for the treatment of small (T1 2) hypopharyngeal squamous cell carcinoma. Clin Exp Otorhinolaryngol 2011;4:44-48.

13 Laccourreye O, Benito J, Ménard M, Garcia D, Malinvaud D, Holsinger FCh. Lateral pharyngotomy in patients with selected squamous cell carcinoma of the lateral oropharynx. Part I : How. Laryngoscope 2013;123:12-17.

14 Laccourreye O, Benito J, Garcia D, Ménard M, Bonfils P, Holsinger FCh. Lateral pharyngotomy for selected invasive squamous cell carcinoma of the lateral oropharynx - Part II : When and Why ? Laryngoscope 2013;123:18-22.

15 Remmert C, Mansour N, Hofauer B' et al. Pharyngotomy in head and neck squamous cell carcinoma: functional and oncological aspects. Acta Otolaryngol 2017; 137:1281-1287.

16 Bertolin A, Ghirardo G, Lionello M, Giacomelli L, Lucioni M, Rizzotto G. Lateral pharyngotomy approach in the treatment of oropharyngeal carcinoma. Eur Arch Otorhinolaryngol_2017;274:2573-2580.

17 Schechter GL, Sly DE, Roper AL $2^{\text {nd }}$, Jackson RT, Bumatay J. Set-back tongue flap for carcinoma of the tongue base. Arch Otolaryngol 1980;106:668-671. 
18 Deganello A, Leemans CR. The infrahyoid flap: a comprehensive review of an often overlooked reconstructive method. Oral Oncol 2014;50:704-710.

19 Rubin F, Laccourreye O, Weisntein GS, Holsinger F Ch. Transoral lateral oropharyngectomy. Eur Ann Otorhinolaryngol Head Neck Dis 2017;134:419-422.

20 Jafari S, Prince RA, Kim DY, Paydarfar D. Sensory regulation of swallowing and airway protection: a role for the internal superior laryngeal nerve in humans. J Physiol 2003;550:287-304.

21 Nguyen NP, Franck MA, Moltz CC, et al. Analysis of factors influencing aspiration risk following chemoradiation for oropharyngeal cancer. Br J Radiol 2009;82:675-680.

22 Shiley SG, Hargunani CA, Skoner JM, Holland JM, Wax MK. Swallowing function after chemoradiation for advanced stage oropharyngeal cancer. Otolaryngol Head Neck Surg 2006;134:455-459.

23 Parsons JT, Mendenhall WM, Stringer SP, et al. Squamous cell carcinoma of the oropharynx: surgery, radiation therapy or both. Cancer 2002;94:2967-2980.

24 Diaz-Molina J, P, Rodrigo JP, Alvarez-Marcos C, Llorente JL, Costales M, Suarez C. Oncological results after surgical treatment of squamous cell cancer of the lateral wall of the oropharynx. Laryngoscope 2011;121:1449-1454. 
Figure 1 : La pharyngotomie latérale de Trotter (tiré de Trotter W. Operations for malignant disease of the pharynx. British Journal of Surgery 1929;16:485-95).

Figure 2 : Illustration du champ opératoire et des principales structures anatomiques avec les quatre éléments vasculo-nerveux (nerf grand hypoglosse, artère linguale, artère thyroïdienne supérieure et nerf laryngé supérieur) qui barrent l'accès au pharynx.

Figure 3 : Illustration des trois principales structures vasculo-nerveuses (nerf grand hypoglosse, artère linguale, nerf laryngé supérieur), les pédicules thyroïdiens et laryngés supérieurs étant liés et sectionnés Le ventre postérieur du muscle digastrique, le muscle mylohyodien et les muscles sous hyoïdiens sont désinsérés respectivement du bord supérieur et inférieur de la grande corne de l'os hyoïde. Les muscles sous hyoïdiens sont séparés de la loge hyothyroépiglottique et du tiers postérieur de la lame cartilagineuse thyroïdienne en incisant le périchondre externe.

Figure 4 : Illustration de l'isolement (pince) des trois principales structures vasculonerveuses (nerf grand hypoglosse, artère linguale, nerf laryngé supérieur) et de la séparation de la paroi pharyngée latérale du plan vasculaire jugulo-carotidien (les ciseaux pointent vers le plan pré vertébral).

Figure 5 : Illustration des trois points d'entrée potentiels (1: trans hyoïdien à la jonction petite corne grande corne de l'os hyoïde, 2: trans cartilagineux thyroïdien, 3: au travers des constricteurs, au bord postérieur de l'aile thyroïdienne). Les pointillés soulignent l'exérèse potentielle de toute la paroi pharyngée latérale, du fond du sinus piriforme en bas, au pôle inférieur de l'amygdale en haut avec à ce niveau la section du muscle styloglosse.

Figure 6 : Dissection anatomique sur sujet frais visualisant une pharyngotomie latérale pour tumeur de la paroi pharyngée postérieure, réalisée au bord postérieur de l'aile thyroïdienne visualisée par deux pinces de Halsted tandis que les ciseaux pointent la paroi pharyngée 
postérieure (1) tout en repoussant le nerf laryngé supérieur (2) isolé en pont et que la pince saisit l'épiglotte. Vers le haut et non isolés, l'artère linguale (3) et le nerf grand hypoglosse (4) qui surcroise les artères carotide interne (5) et externe (6). Le tronc de l'artère thyroïdienne supérieure (7) a été préservé. Le tendon du muscle digastrique (8) n'a pas été désinsérés de la grande corne de l'os hyoïde tout comme les muscles sous hyoïdiens (9) qui ne sont pas décollés de la loge hyothyroépiglottique (10). 


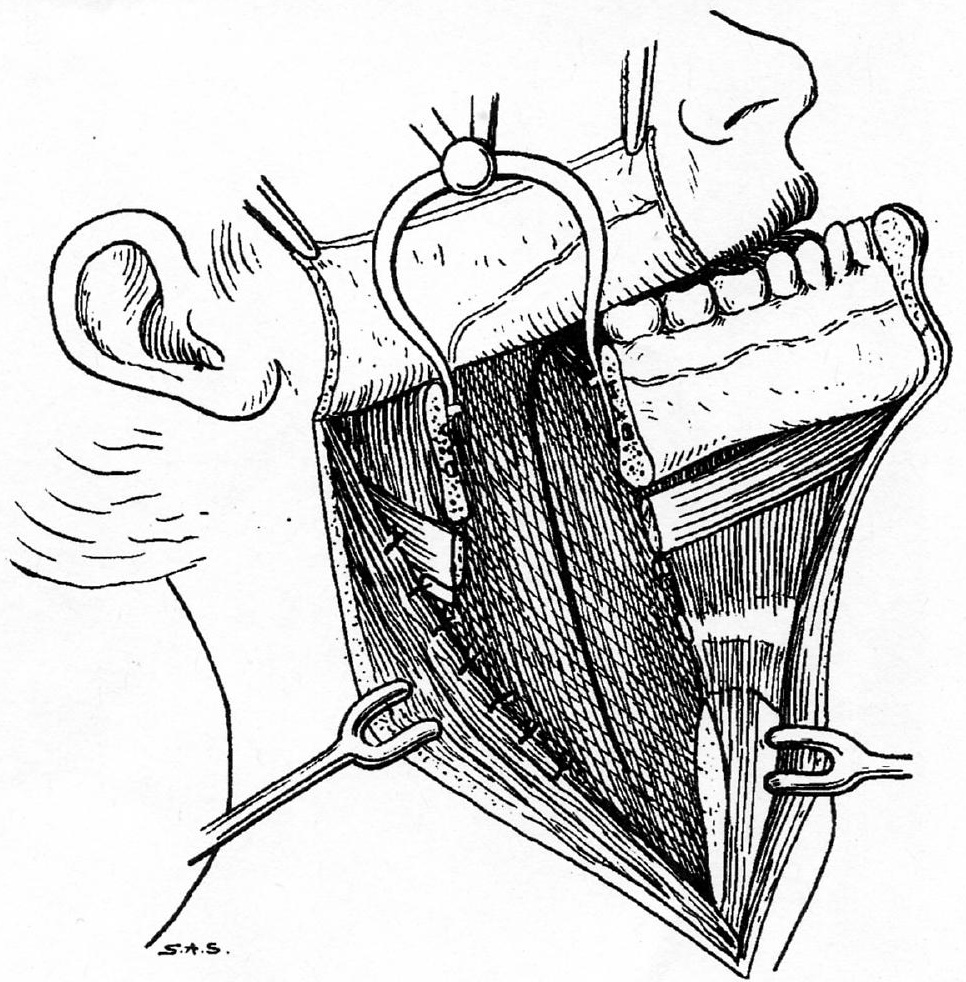




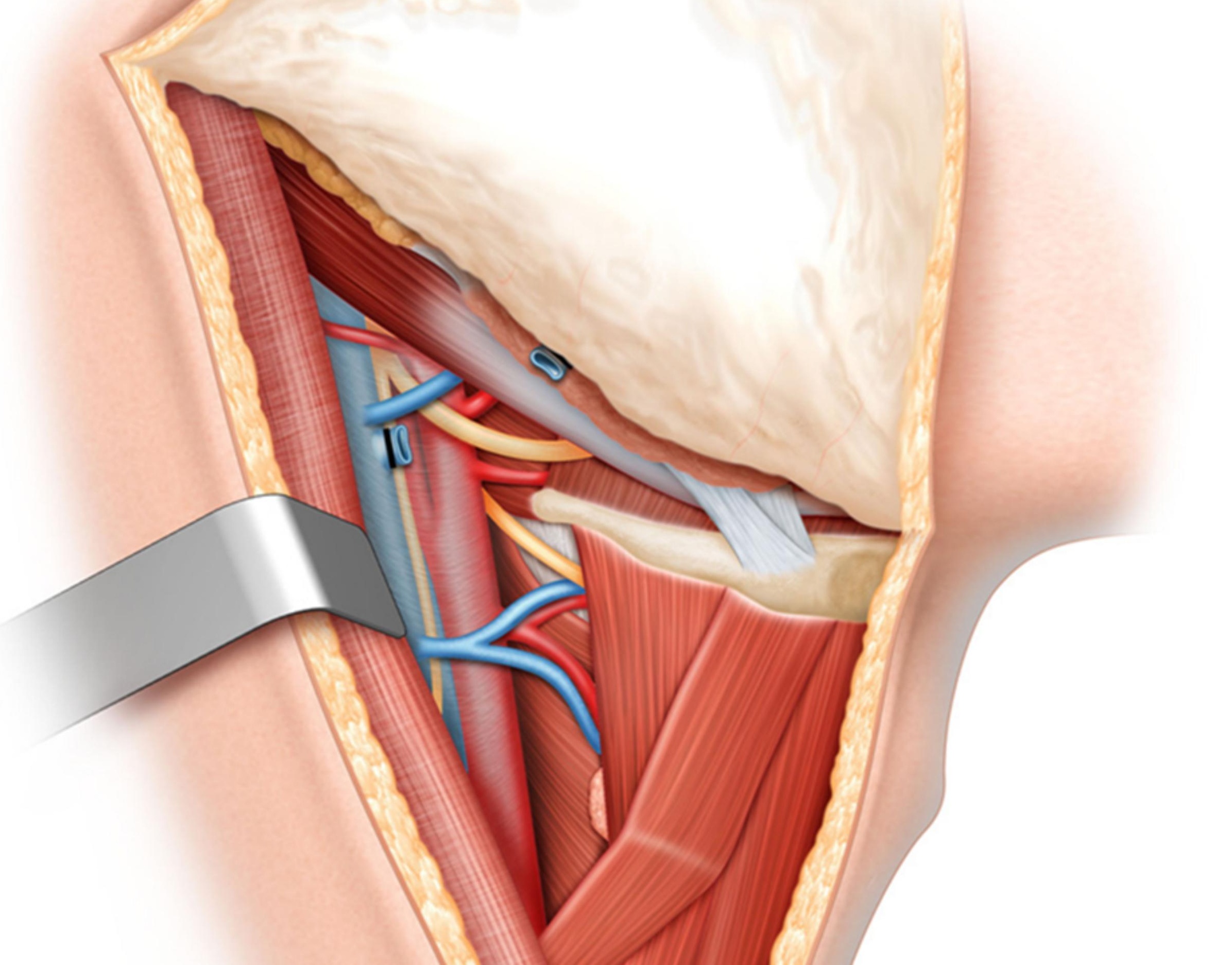




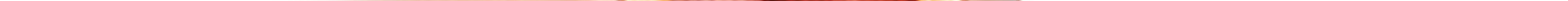




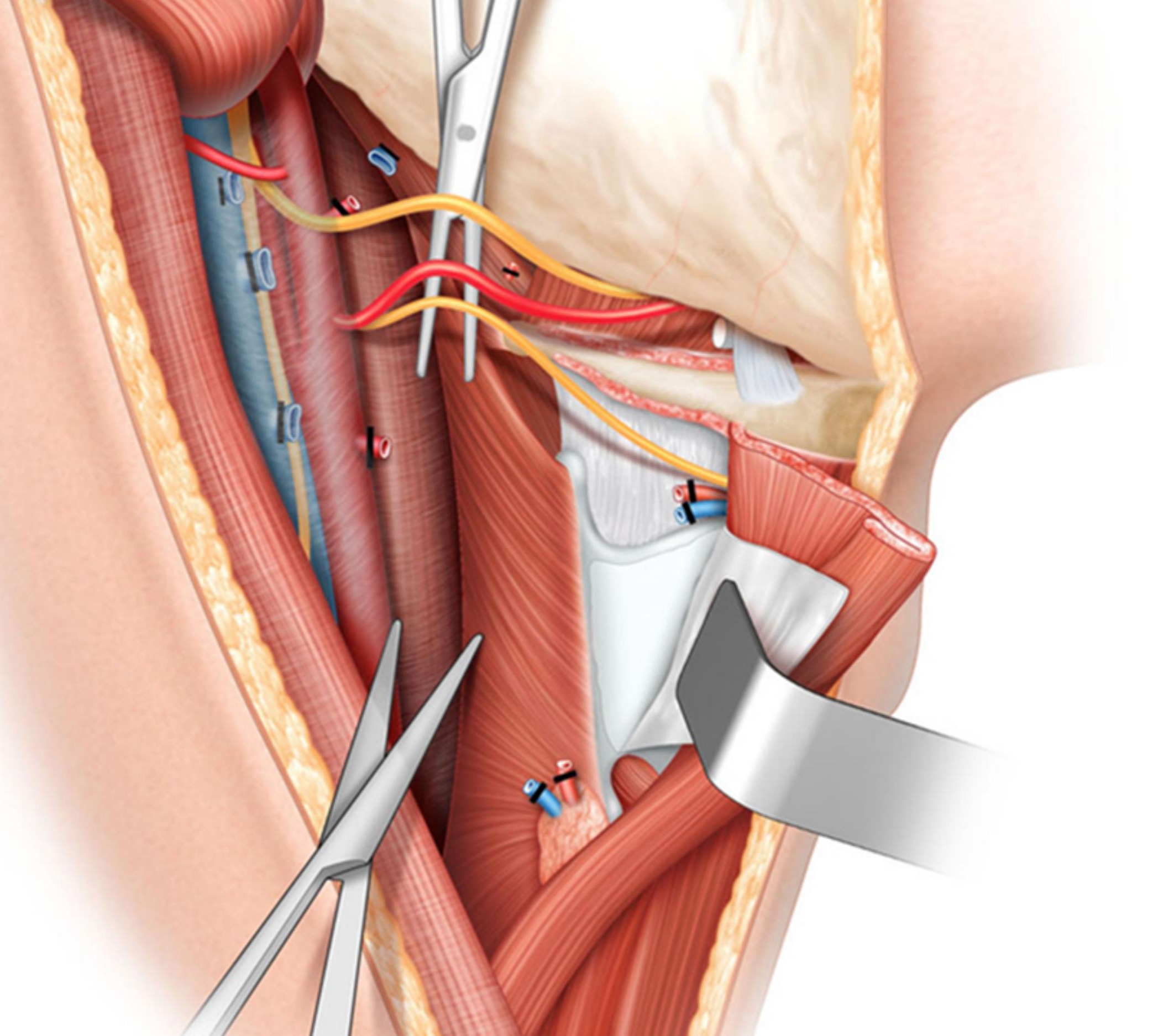




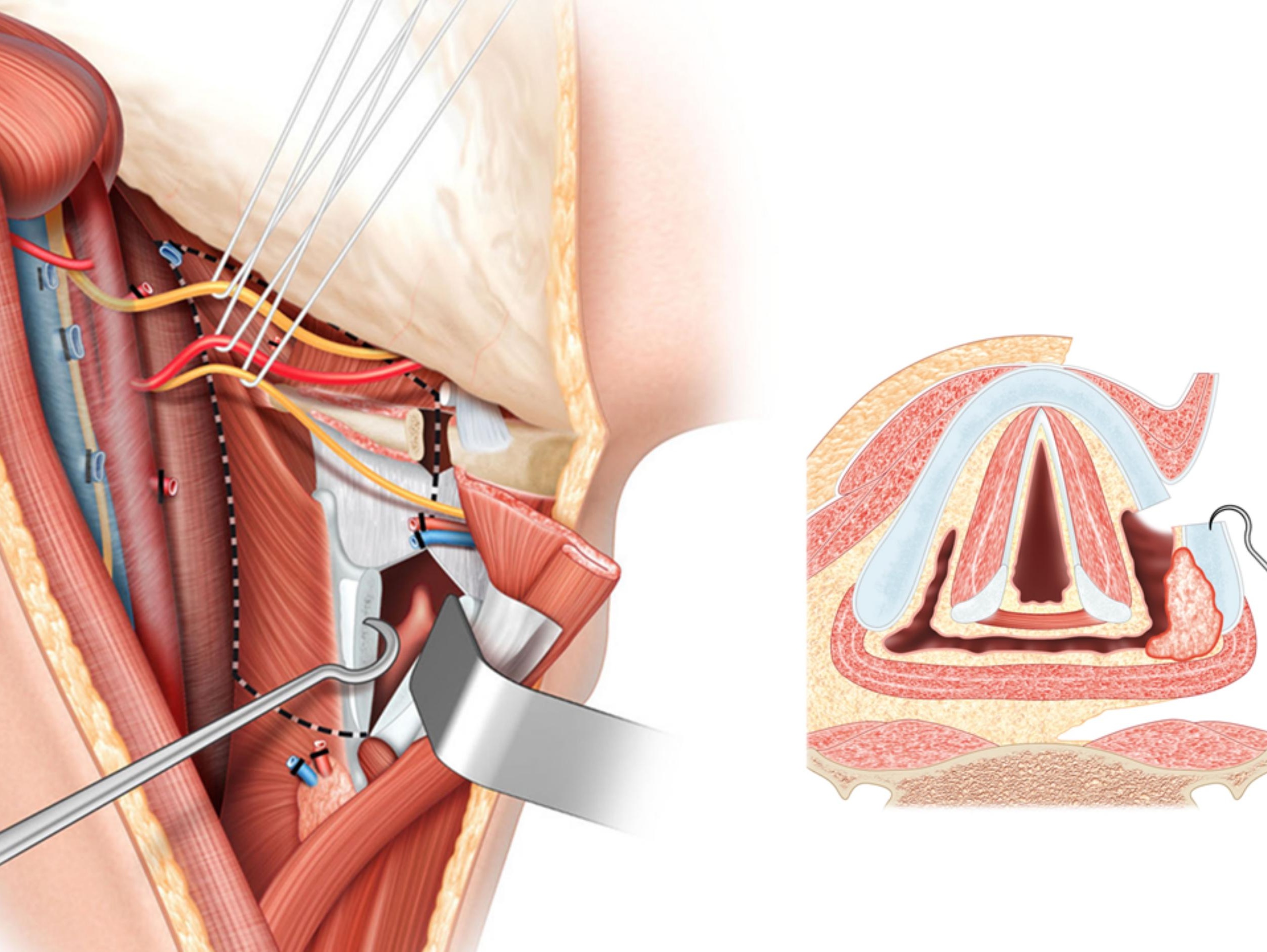




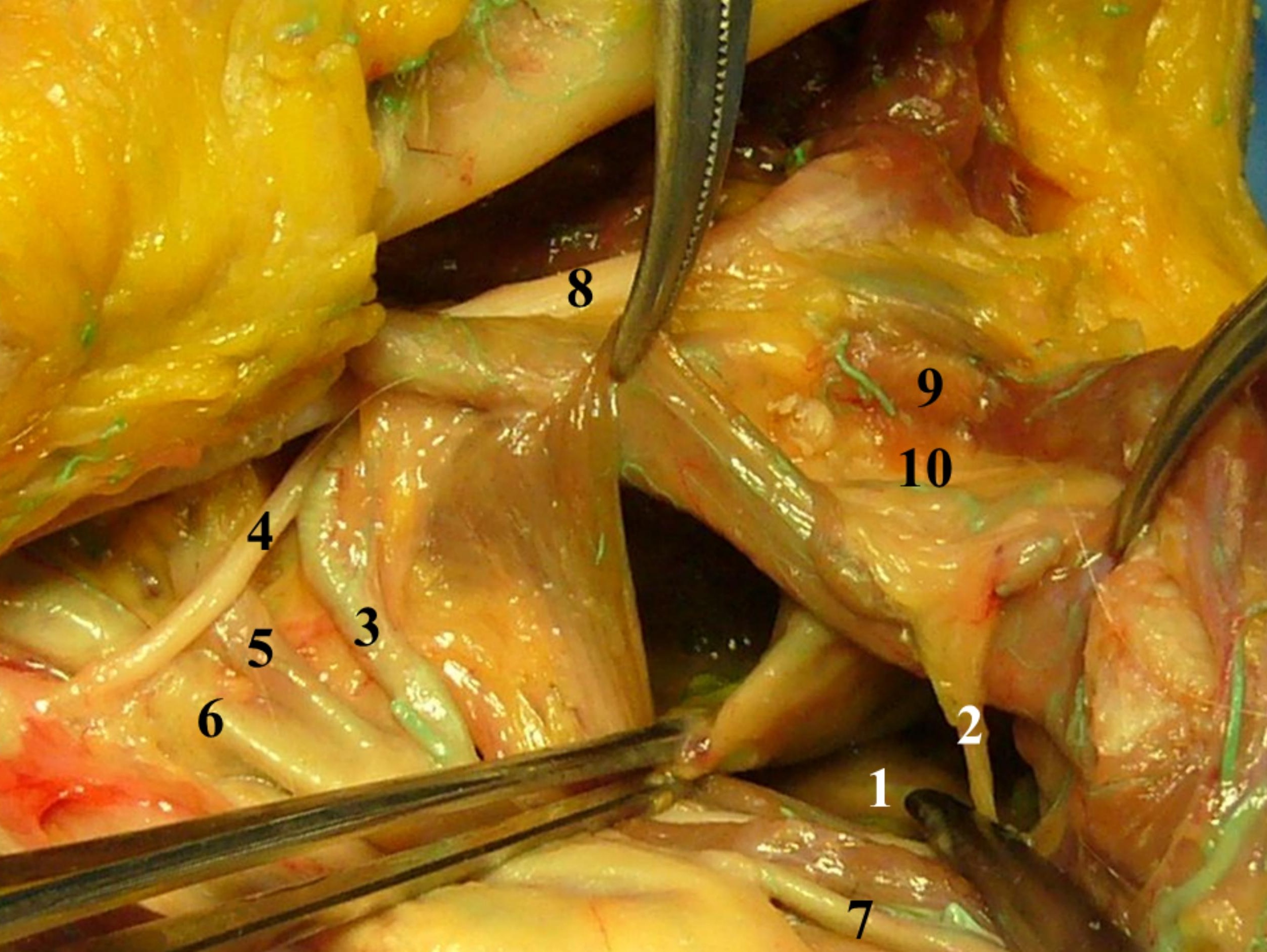

